\title{
Gamma-glutamyl transferase and cardiovascular disease
}

\author{
Gjin Ndrepepa ${ }^{1}$, Adnan Kastrati ${ }^{1,2}$ \\ ${ }^{1}$ Department of Adult Cardiology, Deutsches Herzzentrum München, Technische Universität, Munich, Germany; ${ }^{2}$ DZHK (German Centre for \\ Cardiovascular Research), Partner Site Munich Heart Alliance, Munich, Germany \\ Contributions: (I) Conception and design: G Ndrepepa; (II) Administrative support: None; (III) Provision of study materials or patients: None; (IV) \\ Collection and assembly of data: G Ndrepepa; (V) Data analysis and interpretation: G Ndrepepa; (VI) Manuscript writing: All authors; (VII) Final \\ approval of manuscript: All authors. \\ Correspondence to: Gjin Ndrepepa, MD. Deutsches Herzzentrum, Lazarettstrasse 36, 80636 München, Germany. Email: ndrepepa@dhm.mhn.de.
}

\begin{abstract}
Gamma-glutamyl transferase (GGT) is an enzyme located on the external surface of cellular membranes. GGT contributes in maintaining the physiological concentrations of cytoplasmic glutathione and cellular defense against oxidative stress via cleavage of extracellular glutathione and increased availability of amino acids for its intracellular synthesis. Increased GGT activity is a marker of antioxidant inadequacy and increased oxidative stress. Ample evidence suggests that elevated GGT activity is associated with increased risk of cardiovascular disease (CVD) such as coronary heart disease (CHD), stroke, arterial hypertension, heart failure, cardiac arrhythmias and all-cause and CVD-related mortality. The evidence is weaker for an association between elevated GGT activity and acute ischemic events and myocardial infarction. The risk for CVD or CVD-related mortality mediated by GGT may be explained by the close correlation of GGT with conventional CVD risk factors and various comorbidities, particularly non-alcoholic fatty liver disease, alcohol consumption, oxidative stress, metabolic syndrome, insulin resistance and systemic inflammation. The finding of GGT activity in atherosclerotic plaques and correlation of intra-plaque GGT activity with histological indexes of plaque instability may suggest a participation of GGT in the pathophysiology of CVD, particularly atherosclerosis. However, whether GGT has a direct role in the pathophysiology of CVD or it is an epiphenomenon of coexisting CVD risk factors or comorbidities remains unknown and Hill's criteria of causality relationship between GGT and CVD are not fulfilled. The exploration whether GGT provides prognostic information on top of the information provided by known cardiovascular risk factors regarding the CVD or CVD-related outcome and exploration of molecular mechanisms of GGT involvement in the pathophysiology of CVD and eventual use of interventions to reduce circulating GGT activity remain a duty of future studies.
\end{abstract}

Keywords: Cardiovascular disease (CVD); gamma-glutamyl transferase (GGT); mortality; prognosis

Submitted Oct 20, 2016. Accepted for publication Nov 28, 2016.

doi: $10.21037 /$ atm.2016.12.27

View this article at: http://dx.doi.org/10.21037/atm.2016.12.27

\section{Background information}

Gamma-glutamyl transferase (GGT; EC2.3.2.2.) is an enzyme located on the external surface of membranes of various cells. Mammalian GGT is a dimeric glycoprotein with a molecular weight of $68 \mathrm{kDa}$ consisting of 2 subunits: a $46 \mathrm{kDa}$ large subunit and a $22 \mathrm{kDa}$ small subunit. However, depending on the degree of glycosylation, the molecular weight has been reported to vary between 38 to $72 \mathrm{kDa}$ for the large and 20 to $66 \mathrm{kDa}$ for the small
GGT subunit (1). The large subunit has an intracellular $\mathrm{N}$-terminal sequence, a transmembrane hydrophobic domain and an extracellular domain and is responsible for GGT anchorage on the cellular membrane surface whereas the small subunit harbors the enzyme active center (1). GGT is present in all cells with the exception of erythrocytes. There is considerable inter-tissue and over the embryonic development variability in GGT activity (2). GGT activity was reported to be particularly high in tissues with secretory and absorptive function 


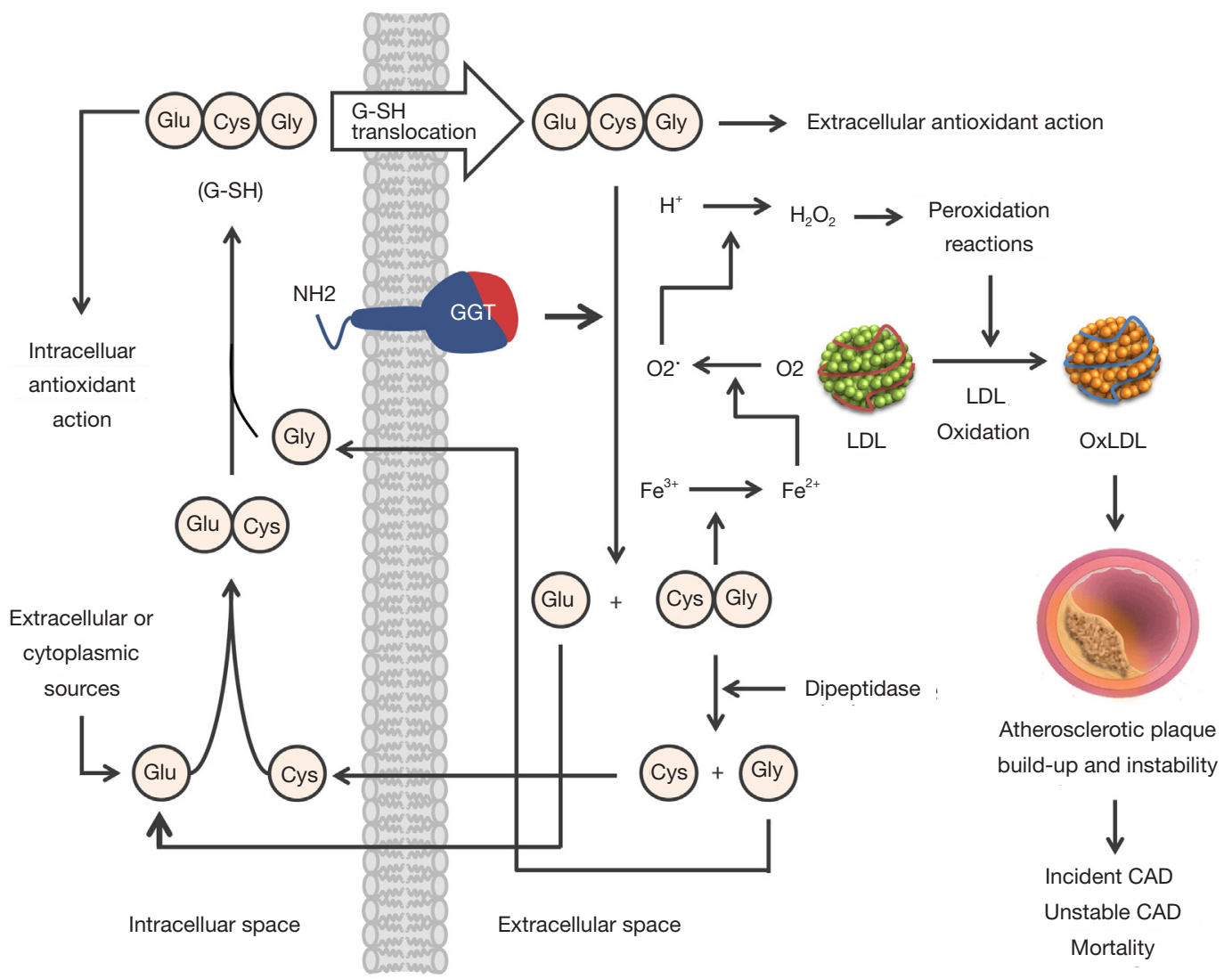

Figure 1 Gamma-glutamyl transferase (GGT) reaction and the proposed mechanism of related pro-oxidant and atherogenic activity. Cys, cysteine; Glu, glutamic acid; Gly, glycine; GSH, glutathione; LDL, low-density lipoprotein; oxLDL, oxidized LDL.

such as kidney, biliary system, intestine and epididymis and the enzyme activity is greatest in the ductal luminal surface of these tissues (3). Abundant GGT activity has been demonstrated in the proximal tubule of the kidney. GGT activity is particularly intensive in biliary pole of hepatocytes and cholangiocytes (4). GGT is produced as a single polypeptide chain which undergoes autoproteolytic cleavage into the large and small subunits. Human GGT is encoded by a multigene family of at least 7 different genes located in the chromosome 22; nevertheless only 1 of these genes produces complete and functional GGT (5). The gene transcription is controlled by multiple promoters. Related gene sequences that are either nonfunctional or represent pseudogenes are found in chromosomes 18, 19, and $20(6,7)$. The multiple promoters and the alternative splicing are responsible for diversity of molecular forms and tissue specificity. Between $50 \%$ and $77 \%$ of GGT activity is genetically determined (8).

GGT functions are not entirely known. The localization of GGT in tissues with transport function has led to the suggestion that GGT is involved in the transport of amino acids via the "gamma-glutamyl cycle" (9). However, the enthusiasm for this hypothesis has faded because humans or animals with GGT deficiency have no anomalies in amino acid transport. Cleavage of glutathione-the main thiol antioxidant in humans-is the most important physiological function of GGT. Glutathione has profound cellular functions including protection from oxidative stress, redox signaling, detoxification of xenobiotics, cellular proliferation, fibrogenesis, nitric oxide metabolism, storage and transport of cysteine, sulphur metabolism and apoptosis (10). Glutathione is synthesized in the cytoplasm of cells via a cycle of reactions proposed by Meister (9). After synthesis, glutathione is transported out of the cell and is degraded by GGT into glutamyl moiety (transferred to water or other compounds such as amino acids or peptides) and dipeptide cysteinyl-glycine which is further degraded by dipeptidase into free cysteine and glycine (Figure 1). 
Glutathione breakdown in the extracellular space increases the availability of cysteine which is taken up by the cells and used as an essential precursor for the intracellular synthesis of glutathione and proteins. Thus GGT contributes in maintaining the physiological concentrations of glutathione in cytoplasm and cellular defense against oxidative stress. GGT deficiency is an extremely rare autosomal recessive disease characterized by increased glutathione concentration in plasma and urine (due to cellular loss not compensated by GGT action) and central nervous system alterations (11). Other functions of GGT include involvement in the metabolism of leukotrienes, xenobiotics and glutaminase action (cleavage of amide bond of amino acid glutamine to produce glutamate and ammonium) (2).

Circulating GGT is supposed to originate mostly from the liver $(12,13)$ and is influenced by genetic and environmental factors (14). Based on gel filtration chromatography, researchers from the Pisa University have identified 4 GGT fractions with different molecular weight in subjects of both sexes: big (b-GGT), medium (m-GGT), small (s-GGT) and free (f-GGT) (15). Recent studies have suggested that b-GGT consists of membrane microvesicles and it may serve as a precursor for smaller fractions (m-GGT and s-GGT) whereas f-GGT represents free soluble form of the enzyme (4). Emerging evidence suggests an association between elevation of various GGT fractions and specific diseases (16); however, this issue needs further investigation. Although GGT measurement was introduced into clinical laboratories more than 50 years ago and research continuously improved our understanding of clinical utility of GGT, questions still remain as regards the indications for GGT measurement, laboratory methods and reference range. The list of GGT involvement in pathological processes is long. Nevertheless, outside the clinical use as a test for hepatobiliary disease and alcohol abuse, GGT has garnered large interest for its association with cardiovascular disease (CVD), diabetes, metabolic syndrome and cancer. The focus of this review is to summarize the existing knowledge on the association of GGT with CVD. The association of GGT with other morbid conditions is not covered. For detailed information, the reader may refer to recent reviews on these topics $(8,17,18)$.

\section{GGT, CVD and mortality}

Several population-based studies have investigated the association of GGT with CVD or risk of death. The Framingham Offspring Study (FOS) was one of the first epidemiological studies initiated to test the association of GGT with cardiovascular risk and disease. In its second examination cycle, 3,451 patients were recruited between 1978 and 1982 and followed up to a mean of 19 years. GGT was associated with higher body mass index, blood pressure, low-density lipoprotein (LDL)-cholesterol, triglycerides and glucose. On the follow-up, per each standard deviation (SD) higher log GGT, the risk for metabolic syndrome increased by $26 \%$. After adjustment for cardiovascular risk factors, GGT conferred a $13 \%$ and $26 \%$ increase in the risk for CVD and mortality for each SD higher log GGT. Subjects in the highest GGT quartile showed a $67 \%$ increase in the incidence of CVD. The study clearly showed that increased activity of circulating GGT predicted the onset of metabolic syndrome, incident CVD and mortality hinting a role for GGT as a marker of metabolic and cardiovascular risk (19). In the British Regional Heart Study-a prospective study of 7613 middle-aged British men followed for 11.5 years-GGT was associated with an array of cardiovascular risk factors, all-cause mortality and mortality related to coronary heart disease (CHD). Increased GGT [highest quintile ( $>24 \mathrm{U} / \mathrm{L}) v s$. the rest] was associated with $22 \%$ and $42 \%$ increase in the relative risk for all-cause and CHD-related mortality. Notably, the risk for CHD-related mortality linked to GGT (a 42\% increase in adjusted risk) was higher for subjects with evidence of CHD, particularly prior myocardial infarction, at the time of screening (20). A prospective study by Wannamethee et al. (21) included 6,997 men, 40-59 years of age, without a history of CVD, stroke or diabetes. Over a 24-year followup, GGT was significantly associated with the increased risk of fatal CHD events (but not non-fatal events), major stroke and overall CVD mortality after adjustment for traditional CVD risk factors. The risk of fatal CHD and CVD-related mortality was elevated in the top quarter ( $\geq 22 \mathrm{IU} / \mathrm{L})$ only, whereas the risk of stroke showed a tendency to increase with raising GGT level. The adjusted relative risks for GGT quartile 4 versus quartile 1 were 1.43 (1.09-1.84), 1.56 (1.20-2.04) and 1.40 (1.16-1.70) for fatal CHD events, stroke and CVD mortality, respectively. A stronger association of GGT with CVD mortality was seen in the men of younger (<55 years) age (21). The British Women's Heart and Health study assessed the association of GGT with CHD or stroke in 4,286 women (2,961 women with completed data) over a 4.6-year follow-up. For each unit higher log GGT, the hazard ratios (HR) with $95 \%$ confidence interval (CI) were 
1.15 (0.88-1.48) for incident CHD, 1.45 (0.90-2.34) for incident stroke and 1.17 (0.93-1.48) for CHD or stroke (22). In the meta-analysis of 10 prospective studies incorporated in the same publication, the HR per $1 \mathrm{U} / \mathrm{L}$ change of GGT was $1.20(1.02-1.40)$ for CHD, $1.54(1.20-2.00)$ for stroke and $1.34(1.22-1.48)$ for CHD or stroke (22).

Three large Austrian studies have investigated the association of GGT with cardiovascular risk factors, CVD or mortality. The study by Kazemi-Shirazi et al. (23) investigated the association of GGT with the risk of mortality in a cohort of 283,438 first attendants (inpatients or outpatients). The median follow-up was 7.6 years. GGT activity was scaled into five categories: normal low $(<9 \mathrm{U} / \mathrm{L}$ for women, $<14 \mathrm{U} / \mathrm{L}$ for men), normal high (9-17, 14-27 U/L), moderately elevated (18-26, 28-41 U/L), increased (27-35, $42-55 \mathrm{U} / \mathrm{L})$, and highly elevated ( $\geq 36, \geq 56 \mathrm{U} / \mathrm{L})$. The adjusted HRs for all-cause mortality were 1.2 (1.1-1.2), $1.4(1.3-1.4), 1.6(1.5-1.6)$ and $2.0(2.0-2.1)$ for 2 nd to 5 th categories versus 1 st (normal low) GGT category, suggesting a dose-response relationship between GGT and the risk of mortality (23). The study by Ruttmann et al. (24) assessed the association of GGT with the risk of CVD-related mortality in a cohort of 163,944 Austrian adults, monitored for up to 17 years. High GGT was significantly associated with CVD-related mortality with adjusted HR per log GGT of $1.66(1.40-1.98)$ in men and 1.64 (1.36-1.97) in women. Subgroup analyses in men showed a significant association of GGT with incident fatal events of chronic forms of CHD, congestive heart failure, hemorrhagic and ischemic stroke but not myocardial infarction $(\mathrm{P}=0.16)$. Comparable associations were also observed in women except for the association with hemorrhagic or ischemic stroke which was not significant. The analyses stratified by age showed stronger associations in younger participants (24). In the study by Strasak et al. (25), a cohort of 76,113 Austrian men and women with serial measurements of GGT was prospectively followed-up for a median of 10.2 years. Longitudinal changes of GGT were assessed over a period of 6.9 years. A 7-year change of GGT of $>9.2 \mathrm{U} / \mathrm{L}$ was associated with increased CVD mortality in men [adjusted HR $=1.40$ (1.09-1.81)] compared with stable GGT (7-year change of -0.7 to $1.3 \mathrm{U} / \mathrm{L})$. In women, the risk of CVD mortality was also increased with increasing GGT, but the effects were less evident and were significant only for CHD. As in the study by Ruttmann et al. (24) markedly stronger associations were observed in younger individuals.

Two prospective studies in Finnish population tested the association of GGT with the risk of stroke or CHD. The prospective cohort study by Jousilahti et al. (26) included 14,874 Finnish men and women, 25 to 64 years of age who participated in a cardiovascular risk factor survey in 1982 or 1987. The study showed that serum GGT activity was associated with the risk of total and ischemic stroke in men and women. During a follow-up of 7 or 12 years (depending on the time of survey), the risk ratios in men and women were 1.45 and 1.48 for total stroke and 1.51 and 1.59 for ischemic stroke (calculated per unit increase in log GGT). The prospective study by Lee et al. (27) included 28,838 Finnish men and women, 25 to 74 years of age. The risk of CHD was assessed in the 25 th, 50th, 75 th, and 90th sex-specific percentiles of serum GGT. After adjustment for traditional cardiovascular risk factors, compared with the lowest GGT category ( $<25$ th percentile), the HR for the association with CHD were 1.15, 1.25, 1.27, and 1.57 in men and 1.03, 1.22, 1.32, and 1.44 in women in other 4 GGT categories (25th-50th, 50th-75th, 75th-90th and $\geq 90$ th percentile; P for trend $<0.01)$. Stronger associations were observed in subjects $<60$ years of age and alcohol drinkers. The strength of association was comparable for non-fatal myocardial infarction and fatal CHD. In subjects with type 2 diabetes, the respective adjusted HRs were 1.29, $1.57,1.88$, and 1.78 ( $\mathrm{P}$ trend $=0.03$, for combined men and women) (27).

The EUROSTROKE nested case-control study showed a significant increase in the age- and sex-adjusted risk for stroke of $26 \%$ per SD $(28.7 \mathrm{IU} / \mathrm{mL})$ higher GGT. The odds of hemorrhagic stroke increased linearly with the increase in GGT activity. The association of GGT with stroke was significantly stronger in non-diabetic subjects compared with subjects with diabetes in whom, no association was found (28). The association of GGT activity with the risk for acute ischemic events was investigated in the population-based MONICA (Monitoring trends and determinants on cardiovascular diseases) Augsburg (Southern Germany) survey conducted between October 1984 and June 1985 (29). The study included 1,878 healthy men (25-64 years) who were free of CHD at baseline. Up to 2002, a total of 150 acute ischemic events of new onset occurred. The HR for the association of GGT with incident myocardial infarction across GGT quartiles $(<13,13$ to $<20$, 20 to $<35$, and $\geq 35 \mathrm{U} / \mathrm{L}$ ) were $1.0,1.84,2.02$, and 3.08 (P for trend $<0.001)$. After adjustment, the HR for incident myocardial infarction was $2.34(1.23-4.44)$ for highest versus lowest GGT quartile. The study strongly suggested that GGT elevation predicts the occurrence of acute ischemic 
events in apparently healthy men (29). The populationbased Study of Health in Pomerania (SHIP) recruited 4,160 subjects (2,044 men and 2,116 women) without hepatitis $\mathrm{B}$ and $\mathrm{C}$ or liver cirrhosis at baseline. Over a median of 7.3 years, 307 subjects died. Elevated GGT was associated with the risk of mortality in men [adjusted HR $=1.49(1.08$ 2.05)] but not in women [adjusted HR $=1.30(0.80-2.12))$ with both risk estimates calculated for 5 th versus 1 st-4th GGT quintiles. The association was particularly strong in men with hepatic steatosis $[\mathrm{HR}=1.98(1.21-3.27)]$. The authors concluded that in subjects with increased GGT levels, ultrasound examination of liver should be performed for diagnostic and risk stratification reasons (30). Other studies have demonstrated an association of GGT with mortality in elderly subjects $(31,32)$.

Two studies in Asian population (Japan) have suggested a stronger association between GGT and CVD-related mortality and stroke in women than in men. The study by Hozawa et al. (33) included 2,724 Japanese men and 4,122 Japanese women without prior CVD or liver dysfunction. Over a follow-up of 9.6 years there were 83 and 82 CVD deaths, in men and women, respectively. In women, the adjusted HR for CVD mortality was 2.88 (1.14-7.28) for the elevated GGT group (GGT $\geq 50 \mathrm{U} / \mathrm{L}$ ) versus the reference group (GGT: 1-12 U/L). No significant association was observed in men. The association remained significant in the never-drinker subgroup (33). Similar findings as regards the gender-specific association of GGT with CVD mortality were reported in the prospective study by Fujiyoshi et al. (34) which included 7,488 adults (3,089 men). The risk for stroke was not increased with the increase in GGT activity either in women or in men (34). As regards the association between GGT and CVD mortality, a 2009 report of the third National Health and Nutrition Examination Study (NHANES III) 1988-1994 Survey that included 14,950 adult participants without viral hepatitis B and $\mathrm{C}$ came to different conclusions. Abnormal GGT was defined as $>51 \mathrm{U} / \mathrm{L}$ in men and $>33 \mathrm{U} / \mathrm{L}$ in women. Death certificate-based 12-year mortality was assessed. All-cause mortality $[\mathrm{HR}=1.5(1.2-1.8)]$ and mortality related to liver disease [HR =13.0 (2.4-71.5)], cancer [HR =1.5 (1.01-2.2)] and diabetes $[\mathrm{HR}=3.3(1.4-7.6)]$ but not CVD mortality $[\mathrm{HR}=1.3(0.8-2.0)]$ increased with the increase in GGT activity (35).

Several recent meta-analyses have summarized the results of studies that have assessed the association of GGT with CVD or mortality. A 2013 meta-analysis of 7 studies with 273,141 participants showed an association between
GGT and cardiovascular [relative risk $=1.52(1.36-1.70)$ ] and all-cause [relative risk $=1.56(1.34-1.83)]$ mortality with both risk estimates calculated for highest versus lowest GGT quartile. Notably the association between GGT and cardiovascular mortality was not significant in the subgroup of Asian participants [relative risk =1.76 (0.76-3.30)] (36). A 2014 meta-analysis by Kunutsor et al. (37) of 11 prospective studies has shown a significant association between elevated GGT and mortality [fully adjusted relative risk $=1.60$ (1.42-1.89) for 3rd versus 1st GGT tertile with substantial heterogeneity between studies]. Another 2014 meta-analysis of 29 cohort studies with 1.23 million participants and 20,406 cardiovascular outcomes showed an association between GGT and CVD with an adjusted relative risk of 1.23 (1.16-1.29) per SD higher log GGT (38). Alkaline phosphatase but not alanine aminotransferase or aspartate aminotransferase showed also an independent association with CVD risk.

In aggregate, evidence from epidemiological studies as regards the association of GGT with the risk for CVD or mortality may be summarized as follows: first, epidemiological evidence strongly supports an association between elevated GGT and incident CVD, stroke or all-cause and CVD-related mortality which appears to be stronger in subjects of younger age. Second, there seems to be a strong correlation between elevated GGT and cardiometabolic risk factors which tend to cluster in subjects with higher GGT levels. Consequently, whether GGT provides prognostic information on top of cardiovascular and metabolic risk factors for prediction of CVD or mortality remains unproven. Third, evidence available is not consistent as regards the association between elevated GGT activity and the risk for acute ischemic events, particularly acute myocardial infarction. Fourth, although a significant association between elevated GGT and CVD or mortality is observed in both sexes, more sex-specific analyses are needed to clarify some existing controversy. Whether the association between GGT and CVD or mortality is weaker in Asian population needs further investigation.

\section{GGT and coronary events or mortality in patients with CHD}

It has been suggested that the presence CHD may strengthen the association between GGT and mortality (20). Emdin et al. (39) assessed the association of GGT with mortality or mortality plus nonfatal myocardial infarction over a 6-year follow-up in 469 patients with angiography- 
documented CHD. In a subgroup of 262 patients with previous myocardial infarction, cardiac mortality $(25.2 \%$ vs. $13.9 \% ; \mathrm{P}=0.038)$ or cardiac mortality plus nonfatal myocardial infarction $(32.7 \%$ vs. $20.4 \% ; \mathrm{P}=0.031)$ were higher in patients with a GGT $>40 \mathrm{U} / \mathrm{L}$ versus those with GGT $<40 \mathrm{U} / \mathrm{L}$. The association between GGT and cardiac events remained significant after adjustment for potential confounders including alcohol consumption. Notably, GGT had no significant prognostic value in patients without previous myocardial infarction (39). In a more recent study, the same authors reconfirmed the independent association of GGT with cardiac mortality in 474 patients with established CHD. At 3 years cardiac mortality was $9 \%$ in patients with GGT $>25 \mathrm{U} / \mathrm{L}$ and $3.5 \%$ in patients with GGT $<25 \mathrm{U} / \mathrm{I}(\mathrm{P}=0.028)$. The combination of three biomarkers (higher GGT, C-reactive protein and fasting glucose) identified a group of patients $(\mathrm{n}=45)$ with a 3 -year risk of cardiac mortality of $26.6 \%$ (40). In the Ludwigshafen Risk and Cardiovascular Health (LURIC) study, GGT was measured in 2,556 subjects with and 699 subjects without angiography-proven CHD. There were 754 deaths over a mean follow-up of 7.75 years. All-cause and CVD-related mortality was significantly increased from 1st to 4th GGT quartiles. Compared with subjects with GGT in the lowest quartile, in other 3 quartiles the HR were 1.2 (0.9-1.5), $1.4(1.1-1.8)$ and $1.9(1.5-2.3)$ for all-cause mortality and 1.4 (1.0-2.0), $1.8(1.4-2.5)$ and $2.2(1.6-2.9)$ for mortality due to cardiovascular causes. In patients with angiographic CHD, the association between GGT and prognosis was significant and comparable to that of the entire cohort (41). Other studies have shown that GGT predicts prognosis in patients with stable CHD (42) or in-hospital major adverse cardiovascular events in patients presenting with ST-segment elevation myocardial infarction (43).

Our group investigated the association of GGT with cardiovascular events in 5,501 consecutive patients with angiography-proven CHD (44) and in a subgroup of 2,534 consecutive patients with acute coronary syndromes (45). A specific aim of our studies was to test whether GGT provides incremental prognostic information beyond that provided by cardiometabolic risk factors in patients with CHD. In patients with GGT in 1st, 2nd and 3rd GGT tertiles (median GGT activity 21.3, 36.6 and 79.6 U/L, respectively), the Kaplan-Meier estimates of 3-year all-cause and cardiac mortality were, $7.1 \%, 7.2 \%$ and $13.9 \%(\mathrm{P}<0.001)$ and $4.1 \%$, $3.6 \%$ and $7.9 \%(\mathrm{P}<0.001)$, respectively. After adjustment, GGT was associated with $30 \%$ and $21 \%$ increase of the risk for all-cause and cardiac mortality, respectively. The
C-statistic of multivariable models was used to test the discriminatory ability of GGT regarding mortality. Of note, GGT improved the discriminatory power of the model(s) of all-cause mortality $(\mathrm{P}<0.001)$ and non-cardiac mortality $(\mathrm{P}<0.001)$ but not cardiac mortality $(\mathrm{P}=0.155)$. This study suggested that GGT provides prognostic information that is incremental to the information provided by CVD risk factors for all-cause and non-cardiac but not cardiac mortality (44). The study in patients with acute coronary syndromes showed comparable results in terms of association of GGT with all-cause mortality (45). Surprisingly, however, adjustment in the multivariable Cox model attenuated the association of GGT with cardiac mortality to the level of statistical insignificance. Discriminatory tests (C-statistic) showed that GGT did not provide prognostic information on top of CVD risk factors regarding prediction of cardiac mortality even in patients with acute coronary syndromes, known to die mostly from cardiac causes (45). In both studies there was no association of GGT with the risk of non-fatal myocardial infarction or stroke $(44,45)$.

Whether GGT correlates with the extent or severity of coronary atherosclerosis remains a matter of controversy. A recent study 442 consecutive patients with stable CHD showed a close correlation between GGT and severity and extent of coronary atherosclerosis assessed by the SYNTAX score (46). Similarly, another recent study that used computed tomography angiography to assess coronary plaque burden and structure in 259 young subjects with coronary atherosclerosis (138 patients with coronary atherosclerosis with a mean age of 41.6 years and 121 controls with a mean age of 41.9 years) showed that patients with plaque formation had significantly higher GGT levels than controls. Furthermore, GGT levels were correlated with the number of plaques and the presence of noncalcified plaques (47). However, other studies came to opposite conclusions. In the study by Saely et al. (48) that included 1,000 patients undergoing coronary angiography, GGT was associated with metabolic syndrome but not with angiographydocumented coronary atherosclerosis. We also did not find any correlation between GGT level and extent of coronary atherosclerosis or proportion of patients with multi-vessel disease, despite showing a strong association of GGT with all-cause and cardiac mortality (44). One study found that the serum level of GGT may be an independent marker for in-stent restenosis (49). Other studies have shown an association of GGT with coronary 
flow reserve in hypertensive patients (50), coronary slow flow (51), no re-flow after primary percutaneous coronary intervention (52), syndrome $X$ (53), carotid intima media thickness (54) or contrast-induced nephropathy in patients with acute myocardial infarction (55).

In aggregate, evidence for an association of GGT with coronary events and mortality in patients with established CHD is weaker than the evidence obtained from epidemiological studies investigating the association of GGT with incident CHD or mortality. Particularly, whether GGT offers prognostic information that is incremental to the information provided by conventional risk factors remains still questionable. Despite using coronary angiography - the gold standard for diagnosis of CHD-studies that assessed GGT in patients with established CHD included smaller numbers of patients and had a short follow-up. Furthermore teasing out the intricate relationship and interdependence between CVD risk factors, GGT and CHD remain difficult. The association of GGT with mortality may be underadjusted, particularly for the presence and impact of non-alcoholic fatty liver disease-a highly prevalent chronic liver disease and an equivalent of metabolic syndrome with an established role in CVD and mortality $(56,57)$. A large Korean population study showed that GGT predicted an increased risk for allcause and cancer-related mortality which persisted after adjustment for liver fat. Nevertheless adjustment for the liver fat attenuated the association of GGT with CVD mortality (58). The confounding impact of percutaneous coronary intervention-suggested to abolish the association of GGT with coronary events (39) — should be considered. Finally the vast majority of contemporary patients with CHD are treated with statins and other secondary prevention measures. Statins are known to reduce the risk of fatal and nonfatal coronary events due to their plaque stabilization effects and as shown for other biomarkers may attenuate the association of GGT with cardiovascular events in patients with CHD. Recent evidence shows that non-cardiac deaths have exceeded cardiac deaths in patients with CHD (59), potentially reflecting the efficacy of secondary prevention measures. An experimental study in apolipoprotein E knockout mice showed that statins significantly reduced the GGT expression in aortic atherosclerotic plaques (60). Not surprisingly, all these factors may lead to attenuation of the association of GGT with coronary events or cardiac mortality in patients with CHD.

\section{GGT and coronary events or mortality in patients with diabetes}

Diabetes and elevated GGT activity are associated with increased oxidative stress, poor metabolic profile, high prevalence of non-alcoholic fatty liver disease and heightened inflammatory burden. Hypothetically diabetic status and elevated GGT may have a cumulative or additive action exacerbating the impact of these conditions and strengthening the association with CVD and mortality. It has been suggested that the association of GGT with mortality may be stronger in patients with diabetes (27). An observational cohort study of 1,952 patients with type 2 diabetes has shown an association of GGT with allcause, cardiovascular and cancer-related mortality, over a mean follow-up of 6.4 years. Adjusted HR for all-cause, cardiovascular and cancer-related mortality was: 1.047 (1.027-1.067), $1.017(0.973-1.064)$ and 1.052 (1.0171.088), each for $10 \mathrm{U} / \mathrm{L}$ increase in GGT activity. Notably, GGT but not alanine aminotransferase was associated with mortality (61). In 1280 patients with diabetes, Sluik et al. (62) showed that subjects in the 4th GGT quartile had a fully adjusted HR of 3.96 (1.74-9.00] for all-cause mortality over 8.2 years of follow-up. The association was particularly evident in former and current smokers, younger subjects, subjects with a higher waist-height ratio and drinkers. Our group assessed the association of GGT with 3-year mortality in 1,448 diabetic patients and angiography-proven CHD after percutaneous coronary intervention. After adjustment, GGT was associated with the risk for all-cause $[\mathrm{HR}=1.25(1.05-1.49)]$ but not cardiac mortality [HR $=1.23(0.96-1.58)]$, with both risk estimates calculated per SD higher log GGT. The differences in C-statistics of the models with and without GGT showed that GGT improved the risk for all-cause $(\mathrm{P}=0.02)$ but not cardiac $(\mathrm{P}=0.135)$ mortality (63). These studies confirmed an association of GGT with mortality; however, by excluding subjects without diabetes, they offer no information whether the GGT-mortality association is stronger in diabetic patients. A recent study by Kengne et al. (64) addressed this issue. The study included a sample of 17,852 subjects ( $3.3 \%$ with diabetes, $n=583$ ) who were followed for 10.1 years. The age and sex-adjusted HR was $1.43(1.13-1.81)$ in subjects with diabetes and 1.27 $(1.18-1.37)$ in subjects without diabetes for CVD mortality and $1.24(1.08-1.44)$ and $1.30(1.25-1.34)$, respectively, for all-cause mortality, with all risk estimates calculated per 1 SD increment in log GGT. Notably no evidence was found 
that diabetic status per se modified the strength of the GGT-mortality association (P for interaction $=0.16)(64)$.

These studies showed that elevated GGT activity predicts the risk for all-cause mortality in diabetic patients. The association of GGT with CVD mortality seems to be weaker than the association with all-cause mortality. There is no solid evidence that diabetic status modifies the association of GGT with mortality.

\section{GGT and heart failure}

Several studies have assessed the association of GGT with incident congestive heart failure. In 2010, Dhingra et al. (65) reported GGT-heart failure relationship in 3,544 participants of the Framingham study cohort who were free of heart failure or myocardial infarction. Over a mean follow-up of 23.6 years, the risk of heart failure was increased by $39 \%$ [adjusted HR $=1.39(1.20-1.62)$ ] per each SD higher log GGT). Subjects with a serum GGT level $\geq$ median had a 1.71 -fold risk of heart failure compared with individuals with GGT < median. Of importance was the finding that GGT improved the risk reclassification modestly (net reclassification index of $5.7 \% ; \mathrm{P}=0.01$ ). The Kuopio Ischaemic Heart Disease (KIHD) study followed 1,780 men free of heart failure for a mean of 22 years (66). Serum GGT was log-linearly associated with the risk of heart failure [adjusted HR $=1.25(1.07-1.45)$ per $1 \mathrm{SD}$ higher $\log$ GGT]. The findings remained consistent in analyses accounting for CHD of new onset and incident impaired renal function. In a meta-analysis of 5 studies (incorporated in the same study) the fully adjusted relative risk for heart failure per each SD higher baseline and longterm GGT values were $1.28(1.20-1.35)$ and 1.43 (1.311.56), respectively (66). These studies strongly support an association of GGT with the risk for heart failure. Some studies suggest a direct participation of GGT in the pathophysiology of congestive heart failure (67).

\section{GGT and arterial hypertension}

Several lines of evidence suggest an association between GGT and arterial hypertension. Cheung et al. (68) showed in 235 patients with arterial hypertension and 708 normotensive subjects that GGT but no other liver enzymes predicted new-onset hypertension over a 3-year follow-up. Other studies have shown that GGT correlates with systolic and diastolic blood pressure (69), arterial stiffness $(69,70)$ or impaired aortic elasticity (71). Elevated but still within normal range GGT correlated with incident hypertension in drinkers and non-overweight subjects (72). Recent metaanalyses summarized the evidence linking elevated GGT level with the risk of arterial hypertension $(73,74)$. A recent meta-analysis by Liu et al. (73) summarized 13 prospective studies with 43,314 participants and 5,280 cases of arterial hypertension. The pooled relative risk of hypertension was 1.94 (1.55-2.43) when comparing the highest versus the lowest GGT categories and $1.23(1.13-1.32)$ per each SD higher log GGT. No significant association was observed for the subgroup with blood pressure $\geq 160 / 95 \mathrm{mmHg}$ and nondrinkers. However, heterogeneity across the studies was significant (73). In another recent meta-analysis of 14 cohort studies with 44,582 participants and 5,270 cases of arterial hypertension, the pooled relative risk of association with hypertension was $1.32(1.23-1.43)$ comparing $3 \mathrm{rd}$ versus 1st GGT tertiles (74). Again, the heterogeneity across the studies was significant. In a pooled dose-response analysis of 10 studies, a linear association between GGT and hypertension risk was found (P for nonlinearity $=0.37$ ). The pooled relative risk of arterial hypertension per 5U/L increment in GGT levels was 1.08 (1.04-1.13) (74). These two meta-analyses showed that baseline circulating GGT is associated with the risk of arterial hypertension in general population, consistent with a linear dose-response relationship.

\section{GGT, cardiac arrhythmias and sudden cardiac death}

The rationale for investigating the association between GGT and cardiac arrhythmias rests on the link of GGT with some well-known factors that predispose for cardiac arrhythmias such as, cardiovascular risk factors, liver disease, alcohol consumption, systemic inflammation and increased oxidative stress. An indirect impact of GGT via other forms of CVD predisposing for cardiac arrhythmias should also be considered. Several studies have addressed the association of GGT with the risk for atrial fibrillation. The Atherosclerosis Risk in Communities (ARIC) study in which 1,021 incident atrial fibrillation events occurred in 9,333 men and women over a 12-year follow-up showed that GGT was linearly associated with the risk for atrial fibrillation after multivariable adjustment. A doubling of GGT activity was associated with $20 \%$ increased risk of atrial fibrillation (75). The SHIP study showed that elevated activities of liver enzymes were associated with increased prevalence of atrial fibrillation in general adult 
population. The adjusted odds ratio for atrial fibrillation was 2.17 (1.64-2.87) per each SD higher log GGT (76). The KIHD study that included 1,780 Finnish men showed an association between baseline and long-term GGT values and the risk of atrial fibrillation over a 22 -year followup. However, after full adjustment for conventional risk factors the association was attenuated to below the level of statistical significance (66). A pooled analysis of ARIC and KIHD studies showed that baseline and long-term GGT predicted the risk of atrial fibrillation with fully adjusted HR of 1.09 (1.02-1.16) and 1.14 (1.03-1.25), respectively, per each SD higher baseline and long-term GGT (66). A recent study by Tekin et al. (77) that included 81 patients with nonvalvular atrial fibrillation reported higher levels of GGT in patients with nonvalvular atrial fibrillation compared with age and sex-matched controls without atrial fibrillation. Furthermore the study showed an independent association between GGT and chronic nonvalvular atrial fibrillation. In another recent study, elevated GGT levels predicted the occurrence of atrial fibrillation following catheter ablation (78).

An association between GGT and the risk for ventricular arrhythmias or sudden cardiac death has also been shown. In the KIHD study, the HR for an association of GGT with the risk of ventricular arrhythmias was 1.37 (1.04-1.80) (66). A recent publication from the KIHD study showed that serum GGT was log-linearly associated with the risk of sudden cardiac death with a HR $=1.30(10-1.54)$ per each SD higher $\log$ GGT (79). The association of GGT with sudden cardiac death remained significant after adjustment for known risk factors, alcohol consumption, resting heart rate, lipids and C-reactive protein $[\mathrm{HR}=1.26(1.05-1.50)]$.

\section{Mechanisms of GGT association with CVD}

Several putative explanations may be offered to explain the association of elevated GGT with the risk for CVD or CVD-related mortality. GGT is closely associated with established cardiovascular risk factors (which tend to cluster in patients with higher GGT level), metabolic syndrome and insulin resistance (18). Elevated GGT may be a marker of antioxidant inadequacy and of increased oxidative stress (17). Circulating GGT levels are also closely correlated with the markers of inflammation and thus its elevated activity may signify a heightened inflammatory state (80). Ample evidence exists that all these conditionscardiovascular risk factors, metabolic syndrome, insulin resistance, increased oxidative stress and systemic inflammation are great promoters of CVD and CVD-related mortality. In this regard, elevated GGT may be considered a marker of increased cardiometabolic stress, in general. The close association of GGT with alcohol consumption may also explain the association of GGT and all-cause mortality (81) and cardiac arrhythmias, particularly atrial fibrillation (82). Nevertheless, light and moderate alcohol consumption are inversely associated with myocardial infarction and CVD mortality (81). Nonalcoholic fatty liver-the most common cause of chronic liver disease, with a prevalence up to $70 \%$ in diabetic patients-is associated with the increased activity of GGT and other liver enzymes in addition to more prevalent CVD (83). Nonalcoholic fatty liver disease is associated with an array of cardiometabolic disorders, for which it is considered a metabolic syndrome equivalent. It has been proposed that GGT may indicate a link between fatty liver and early atherosclerotic disease (84). Not surprisingly, the adjustment for fatty liver has attenuated the association between GGT and CVD mortality (58). Nonalcoholic fatty liver disease is associated with the risk of ventricular arrhythmias (85) and atrial fibrillation (86). In particular, elevated GGT is associated with the increased risk for atrial fibrillation either because both conditions share similar risk factors or because of ectopic fat accumulation in atrial myocardium (87) which may modulate the electrophysiological properties and ion currents predisposing for arrhythmogenesis (88). Finally, evidence available suggests a direct participation of GGT in the pathophysiology of CVD, particularly atherosclerosis, on cellular and molecular level. The breakdown of glutathione by GGT in the extracellular space leads to production of cysteinyl-glycine dipeptide-even a stronger reducing agent than glutathione. The cysteinyl-glycine moiety acts as a strong reducing agent of iron from $\mathrm{Fe}^{3+}$ to $\mathrm{Fe}^{2+}$ which subsequently catalyzes formation of superoxide and hydrogen peroxide. These species promote peroxidation reactions (including low-density lipoproteins) and exert a local pro-oxidant and proinflammatory action (Figure 1). There is evidence that these reactions occur within atherosclerotic plaques and they present the most accepted putative mechanism of a direct participation of GGT in the pathophysiology of atherosclerosis leading to promotion of atherosclerotic process, plaque instability and coronary ischemic events (89). Catalytically active GGT is found in atherosclerotic plaques (90) and the GGT activity within the atherosclerotic plaques was found to correlate with systemic GGT activity (91) and histological indexes of plaque instability (92). 


\section{Concluding remarks and perspective}

Ample evidence from large epidemiological studies strongly suggests the existence of an association of elevated GGT activity with CVD, CHD, arterial hypertension, congestive heart failure, cardiac arrhythmias and CVD-related mortality. However, the evidence linking elevated GGT with acute ischemic events is weaker and inconsistent. Although, it has been suggested that GGT appears to pass the Vasan's criteria (93) as a biomarker of increased cardiovascular risk (89), the intricate association of GGT with cardiovascular risk factors and comorbidities, particularly the liver disease, raises serious questions regarding a direct role of GGT in the pathophysiology of CVD or causality in the GGT-CVD relationship. Recent data suggested that adding GGT to conventional cardiovascular risk factors did not improve the risk prediction for CVD or CHD-related mortality $(44,64,94)$. Thus, at this stage, the crucial question whether GGT has a direct role in the pathophysiology of CVD or it is an epiphenomenon of coexisting CVD risk factors or comorbidities remains unanswered. Consequently, the Hill's criteria (95) of causality relationship between GGT and CVD, are not entirely fulfilled. Future studies with the use of specific discriminatory tests to assess whether GGT provides information that is additive and beyond the information provided by known cardiometabolic risk factors as regards CVD or CVD-related outcome are hoped to resolve this dilemma. Exploration of molecular mechanisms of GGT involvement in the pathophysiology of CVD and eventual use of interventions to reduce circulating GGT activity remain a duty of future studies.

\section{Acknowledgements}

None.

\section{Footnote}

Conflicts of Interest: The authors have no conflicts of interest to declare.

\section{References}

1. Castellano I, Merlino A. $\gamma$-Glutamyltranspeptidases: sequence, structure, biochemical properties, and biotechnological applications. Cell Mol Life Sci 2012;69:3381-94.
2. Whitfield JB. Gamma glutamyl transferase. Crit Rev Clin Lab Sci 2001;38:263-355.

3. Castellano I, Merlino A. Gamma-Glutamyl Transpeptidases: Structure and Function. Springer Briefs in Biochemistry and Molecular Biology 2013;VIII:1-57.

4. Fornaciari I, Fierabracci V, Corti A, et al. Gammaglutamyltransferase fractions in human plasma and bile: characteristic and biogenesis. PLoS One 2014;9:e88532.

5. Pawlak A, Lahuna O, Bulle F, et al. gamma-Glutamyl transpeptidase: a single copy gene in the rat and a multigene family in the human genome. J Biol Chem 1988;263:9913-6.

6. Collins JE, Mungall AJ, Badcock KL, et al. The organization of the gamma-glutamyl transferase genes and other low copy repeats in human chromosome 22q11. Genome Res 1997;7:522-31.

7. Figlewicz DA, Delattre O, Guellaen G, et al. Mapping of human gamma-glutamyl transpeptidase genes on chromosome 22 and other human autosomes. Genomics 1993;17:299-305.

8. Kunutsor SK. Gamma-glutamyltransferase-friend or foe within? Liver Int 2016;36:1723-34.

9. Meister A. On the enzymology of amino acid transport. Science 1973;180:33-9.

10. Foyer CH, Noctor G. Redox homeostasis and antioxidant signaling: a metabolic interface between stress perception and physiological responses. Plant Cell 2005;17:1866-75.

11. Ristoff E, Larsson A. Patients with genetic defects in the gamma-glutamyl cycle. Chem Biol Interact 1998;111112:113-21.

12. Shaw LM, London JW, Petersen LE. Isolation of gammaglutamyltransferase from human liver, and comparison with the enzyme from human kidney. Clin Chem 1978;24:905-15.

13. Wenham PR, Horn DB, Smith AF. In vitro studies upon the release of gamma-glutamyltransferase from human liver. Clin Chim Acta 1986;160:223-33.

14. Bathum L, Petersen HC, Rosholm JU, et al. Evidence for a substantial genetic influence on biochemical liver function tests: results from a population-based Danish twin study. Clin Chem 2001;47:81-7.

15. Franzini $M$, Bramanti E, Ottaviano V, et al. A high performance gel filtration chromatography method for gamma-glutamyltransferase fraction analysis. Anal Biochem 2008;374:1-6.

16. Franzini M, Fornaciari I, Fierabracci V, et al. Accuracy of b-GGT fraction for the diagnosis of non-alcoholic fatty liver disease. Liver Int 2012;32:629-34. 
17. Koenig G, Seneff S. Gamma-Glutamyltransferase: A Predictive Biomarker of Cellular Antioxidant Inadequacy and Disease Risk. Dis Markers 2015;2015:818570.

18. Bulusu S, Sharma M. What does serum $\gamma$-glutamyltransferase tell us as a cardiometabolic risk marker? Ann Clin Biochem 2016;53:312-32.

19. Lee DS, Evans JC, Robins SJ, et al. Gamma glutamyl transferase and metabolic syndrome, cardiovascular disease, and mortality risk: the Framingham Heart Study. Arterioscler Thromb Vasc Biol 2007;27:127-33.

20. Wannamethee G, Ebrahim S, Shaper AG. Gammaglutamyltransferase: determinants and association with mortality from ischemic heart disease and all causes. Am J Epidemiol 1995;142:699-708.

21. Wannamethee SG, Lennon L, Shaper AG. The value of gamma-glutamyltransferase in cardiovascular risk prediction in men without diagnosed cardiovascular disease or diabetes. Atherosclerosis 2008;201:168-75.

22. Fraser A, Harris R, Sattar N, et al. Gammaglutamyltransferase is associated with incident vascular events independently of alcohol intake: analysis of the British Women's Heart and Health Study and MetaAnalysis. Arterioscler Thromb Vasc Biol 2007;27:2729-35.

23. Kazemi-Shirazi L, Endler G, Winkler S, et al. Gamma glutamyltransferase and long-term survival: is it just the liver? Clin Chem 2007;53:940-6.

24. Ruttmann E, Brant LJ, Concin H, et al. Gammaglutamyltransferase as a risk factor for cardiovascular disease mortality: an epidemiological investigation in a cohort of 163,944 Austrian adults. Circulation 2005;112:2130-7.

25. Strasak AM, Kelleher CC, Klenk J, et al. Longitudinal change in serum gamma-glutamyltransferase and cardiovascular disease mortality: a prospective populationbased study in 76,113 Austrian adults. Arterioscler Thromb Vasc Biol 2008;28:1857-65.

26. Jousilahti P, Rastenyte D, Tuomilehto J. Serum gammaglutamyl transferase, self-reported alcohol drinking, and the risk of stroke. Stroke 2000;31:1851-5.

27. Lee DH, Silventoinen K, Hu G, et al. Serum gammaglutamyltransferase predicts non-fatal myocardial infarction and fatal coronary heart disease among 28,838 middle-aged men and women. Eur Heart J 2006;27:2170-6.

28. Bots ML, Salonen JT, Elwood PC, et al. Gammaglutamyltransferase and risk of stroke: the EUROSTROKE project. J Epidemiol Community Health 2002;56 Suppl 1:i25-9.

29. Meisinger C, Döring A, Schneider A, et al. Serum gamma- glutamyltransferase is a predictor of incident coronary events in apparently healthy men from the general population. Atherosclerosis 2006;189:297-302.

30. Haring R, Wallaschofski H, Nauck M, et al. Ultrasonographic hepatic steatosis increases prediction of mortality risk from elevated serum gamma-glutamyl transpeptidase levels. Hepatology 2009;50:1403-11.

31. Loomba R, Doycheva I, Bettencourt R, et al. Serum $\gamma$-glutamyltranspeptidase predicts all-cause, cardiovascular and liver mortality in older adults. J Clin Exp Hepatol 2013;3:4-11.

32. Koehler EM, Sanna D, Hansen BE, et al. Serum liver enzymes are associated with all-cause mortality in an elderly population. Liver Int 2014;34:296-304.

33. Hozawa A, Okamura T, Kadowaki T, et al. gammaGlutamyltransferase predicts cardiovascular death among Japanese women. Atherosclerosis 2007;194:498-504.

34. Fujiyoshi A, Miura K, Hozawa A, et al. $\gamma$-Glutamyltransferase and mortality risk from heart disease and stroke in Japanese men and women: NIPPON DATA90. CVD Prevention and Control 2010;5:27-34.

35. Ruhl CE, Everhart JE. Elevated serum alanine aminotransferase and gamma-glutamyltransferase and mortality in the United States population. Gastroenterology 2009;136:477-85.e11.

36. Du G, Song Z, Zhang Q. Gamma-glutamyltransferase is associated with cardiovascular and all-cause mortality: a meta-analysis of prospective cohort studies. Prev Med 2013;57:31-7.

37. Kunutsor SK, Apekey TA, Seddoh D, et al. Liver enzymes and risk of all-cause mortality in general populations: a systematic review and meta-analysis. Int J Epidemiol 2014;43:187-201.

38. Kunutsor SK, Apekey TA, Khan H. Liver enzymes and risk of cardiovascular disease in the general population: a meta-analysis of prospective cohort studies. Atherosclerosis 2014;236:7-17.

39. Emdin M, Passino C, Michelassi C, et al. Prognostic value of serum gamma-glutamyl transferase activity after myocardial infarction. Eur Heart J 2001;22:1802-7.

40. Emdin M, Passino C, Michelassi C, et al. Additive prognostic value of gamma-glutamyltransferase in coronary artery disease. Int J Cardiol 2009;136:80-5.

41. Stojakovic T, Scharnagl H, Trauner M, et al. Serum gamma-glutamyl transferase and mortality in persons undergoing coronary angiography-The Ludwigshafen Risk and Cardiovascular Health Study. Atherosclerosis 2010 Feb;208(2):564-71. 
42. Breitling LP, Grandi NC, Hahmann H, et al. Gammaglutamyltransferase and prognosis in patients with stable coronary heart disease followed over 8 years. Atherosclerosis 2010;210:649-55.

43. Akpek M, Elcik D, Kalay N, et al. The prognostic value of serum gamma glutamyl transferase activity on admission in patients with STEMI undergoing primary PCI. Angiology 2012;63:579-85.

44. Ndrepepa G, Braun S, Schunkert H, et al. Gammaglutamyl transferase and prognosis in patients with coronary artery disease. Clin Chim Acta 2016;452:155-60.

45. Ndrepepa G, Braun S, Cassese S, et al. Relation of Gamma-Glutamyl Transferase to Cardiovascular Events in Patients With Acute Coronary Syndromes. Am J Cardiol 2016;117:1427-32.

46. Aksakal E, Tanboga IH, Kurt M, et al. The relation of serum gamma-glutamyl transferase levels with coronary lesion complexity and long-term outcome in patients with stable coronary artery disease. Atherosclerosis 2012;221:596-601.

47. Celik O, Cakmak HA, Satilmis S, et al. The relationship between gamma-glutamyl transferase levels and coronary plaque burdens and plaque structures in young adults with coronary atherosclerosis. Clin Cardiol 2014;37:552-7.

48. Saely CH, Vonbank A, Rein P, et al. Alanine aminotransferase and gamma-glutamyl transferase are associated with the metabolic syndrome but not with angiographically determined coronary atherosclerosis. Clin Chim Acta 2008;397:82-6.

49. Ulus T, Yildirir A, Demirtas S, et al. Serum gammaglutamyl transferase activity: a new marker for stent restenosis? Atherosclerosis 2007;195:348-53.

50. Caliskan M, Erdogan D, Gullu H, et al. Association between serum gamma-glutamyltransferase levels and coronary microvascular function in hypertensive patients. J Hypertens 2007;25:2497-503.

51. Sen N, Ozlü MF, Başar N, et al. Relationship between elevated serum gamma-glutamyltransferase activity and slow coronary flow. Turk Kardiyol Dern Ars 2009;37:168-73.

52. Ozcan F, Karakas MF, Ozlu MF, et al. Effect of serum gamma-glutamyl transferase levels on myocardial perfusion and long-term prognosis after primary angioplasty in patients with acute ST-elevation myocardial infarction. J Investig Med 2012;60:1186-93.

53. Demir B, Temizhan A, Keskin G, et al. Comparison of serum gamma-glutamyltransferase levels between patients with cardiac syndrome $\mathrm{X}$ and healthy asymptomatic individuals. Kardiol Pol 2012;70:31-7.
54. Yagmur J, Ermis N, Acikgoz N, et al. Elevated serum gamma-glutamyl transferase activity in patients with cardiac syndrome $\mathrm{X}$ and its relationship with carotid intima media thickness. Acta Cardiol 2010;65:515-9.

55. Oksuz F, Yarlioglues M, Cay S, et al. Predictive Value of Gamma-Glutamyl Transferase Levels for ContrastInduced Nephropathy in Patients With ST-Segment Elevation Myocardial Infarction Who Underwent Primary Percutaneous Coronary Intervention. Am J Cardiol 2015;116:711-6.

56. Lonardo A, Ballestri S, Marchesini G, et al. Nonalcoholic fatty liver disease: a precursor of the metabolic syndrome. Dig Liver Dis 2015;47:181-90.

57. Vanni E, Bugianesi E, Kotronen A, et al. From the metabolic syndrome to NAFLD or vice versa? Dig Liver Dis 2010;42:320-30.

58. Sung KC, Ryu S, Kim BS, et al. $\gamma$-Glutamyl Transferase Is Associated with Mortality Outcomes Independently of Fatty Liver. Clin Chem 2015;61:1173-81.

59. Spoon DB, Psaltis PJ, Singh M, et al. Trends in cause of death after percutaneous coronary intervention. Circulation 2014;129:1286-94.

60. Li G, Wu XW, Lu WH, et al. Effect of atorvastatin on the expression of gamma-glutamyl transferase in aortic atherosclerotic plaques of apolipoprotein E-knockout mice. BMC Cardiovasc Disord 2014;14:145.

61. Monami M, Balzi D, Lamanna C, et al. Prognostic value of serum liver enzymes levels in type 2 diabetic patients. Diabetes Metab Res Rev 2007;23:625-30.

62. Sluik D, Beulens JW, Weikert C, et al. Gammaglutamyltransferase, cardiovascular disease and mortality in individuals with diabetes mellitus. Diabetes Metab Res Rev 2012;28:284-8.

63. Ndrepepa G, Colleran R, Luttert A, et al. Prognostic value of gamma-glutamyl transferase in patients with diabetes mellitus and coronary artery disease. Clin Biochem 2016;49:1127-32.

64. Kengne AP, Czernichow S, Stamatakis E, et al. Gammaglutamyltransferase and risk of cardiovascular disease mortality in people with and without diabetes: pooling of three British Health Surveys. J Hepatol 2012;57:1083-9.

65. Dhingra R, Gona $\mathrm{P}$, Wang TJ, et al. Serum gamma-glutamyl transferase and risk of heart failure in the community. Arterioscler Thromb Vasc Biol 2010;30:1855-60.

66. Kunutsor SK, Laukkanen JA, Bluemke DA, et al. Baseline and long-term gamma-glutamyltransferase, heart failure and cardiac arrhythmias in middle-aged Finnish men: Prospective study and pooled analysis of published 
evidence. Eur J Prev Cardiol 2016;23:1354-62.

67. Jiang $\mathrm{S}$, Jiang $\mathrm{D}$, Tao Y. Role of gammaglutamyltransferase in cardiovascular diseases. Exp Clin Cardiol 2013;18:53-6.

68. Cheung BM, Ong KL, Tso AW, et al. Gamma-glutamyl transferase level predicts the development of hypertension in Hong Kong Chinese. Clin Chim Acta 2011;412:1326-31.

69. Jung $\mathrm{CH}, \mathrm{Yu} \mathrm{JH}$, Bae SJ, et al. Serum gammaglutamyltransferase is associated with arterial stiffness in healthy individuals. Clin Endocrinol (Oxf) 2011;75:328-34.

70. Saijo Y, Utsugi M, Yoshioka E, et al. The relationship of gamma-glutamyltransferase to C-reactive protein and arterial stiffness. Nutr Metab Cardiovasc Dis 2008;18:211-9.

71. Celik T, Yuksel UC, Kilic S, et al. The relationship of gamma-glutamyltransferase to aortic elastic properties in young patients with prehypertension. Clin Exp Hypertens 2010;32:377-84.

72. Kim NH, Huh JK, Kim BJ, et al. Serum gamma-glutamyl transferase level is an independent predictor of incident hypertension in Korean adults. Clin Exp Hypertens 2012;34:402-9.

73. Liu CF, Gu YT, Wang HY, et al. Gammaglutamyltransferase level and risk of hypertension: a systematic review and meta-analysis. PLoS One 2012;7:e48878.

74. Kunutsor SK, Apekey TA, Cheung BM. Gammaglutamyltransferase and risk of hypertension: a systematic review and dose-response meta-analysis of prospective evidence. J Hypertens 2015;33:2373-81.

75. Alonso A, Misialek JR, Amiin MA, et al. Circulating levels of liver enzymes and incidence of atrial fibrillation: the Atherosclerosis Risk in Communities cohort. Heart 2014;100:1511-6.

76. Markus MR, Meffert PJ, Baumeister SE, et al. Association between hepatic steatosis and serum liver enzyme levels with atrial fibrillation in the general population: The Study of Health in Pomerania (SHIP). Atherosclerosis 2016;245:123-31.

77. Tekin G, Tekin YK, Senarslan DA, et al. Serum $\gamma$-glutamyltransferase activity in patients with nonvalvular atrial fibrillation. Angiology 2013;64:157-60.

78. Uçar FM, Gucuk İpek E, Acar B, et al. Gamma-glutamyl transferase predicts recurrences of atrial fibrillation after catheter ablation. Acta Cardiol 2016;71:205-10.

79. Kunutsor SK, Khan H, Laukkanen JA. $\gamma$-Glutamyltransferase and Risk of Sudden Cardiac Death in Middle-Aged Finnish Men: A New Prospective Cohort
Study. J Am Heart Assoc 2016;5(2). pii: e002858.

80. Lee DH, Jacobs DR Jr. Association between serum gamma-glutamyltransferase and C-reactive protein. Atherosclerosis 2005;178:327-30.

81. Smyth A, Teo KK, Rangarajan S, et al. Alcohol consumption and cardiovascular disease, cancer, injury, admission to hospital, and mortality: a prospective cohort study. Lancet 2015;386:1945-54.

82. Larsson SC, Drca N, Wolk A. Alcohol consumption and risk of atrial fibrillation: a prospective study and doseresponse meta-analysis. J Am Coll Cardiol 2014;64:281-9.

83. Targher G, Bertolini L, Padovani R, et al. Prevalence of nonalcoholic fatty liver disease and its association with cardiovascular disease among type 2 diabetic patients. Diabetes Care 2007;30:1212-8.

84. Kozakova M, Palombo C, Eng MP, et al. Fatty liver index, gamma-glutamyltransferase, and early carotid plaques. Hepatology 2012;55:1406-15.

85. Mantovani A, Rigamonti A, Bonapace S, et al. Nonalcoholic Fatty Liver Disease Is Associated With Ventricular Arrhythmias in Patients With Type 2 Diabetes Referred for Clinically Indicated 24-Hour Holter Monitoring. Diabetes Care 2016;39:1416-23.

86. Targher G, Mantovani A, Pichiri I, et al. Non-alcoholic fatty liver disease is associated with an increased prevalence of atrial fibrillation in hospitalized patients with type 2 diabetes. Clin Sci (Lond) 2013;125:301-9.

87. Targher G, Valbusa F, Bonapace S, et al. Non-alcoholic fatty liver disease is associated with an increased incidence of atrial fibrillation in patients with type 2 diabetes. PLoS One 2013;8:e57183.

88. Lin YK, Chen YC, Chen JH, et al. Adipocytes modulate the electrophysiology of atrial myocytes: implications in obesity-induced atrial fibrillation. Basic Res Cardiol 2012;107:293.

89. Mason JE, Starke RD, Van Kirk JE. Gamma-glutamyl transferase: a novel cardiovascular risk biomarker. Prev Cardiol 2010;13:36-41.

90. Paolicchi A, Emdin M, Ghliozeni E, et al. Images in cardiovascular medicine. Human atherosclerotic plaques contain gamma-glutamyl transpeptidase enzyme activity. Circulation 2004;109:1440.

91. Franzini M, Corti A, Martinelli B, et al. Gammaglutamyltransferase activity in human atherosclerotic plaques--biochemical similarities with the circulating enzyme. Atherosclerosis 2009;202:119-27.

92. Pucci A, Franzini M, Matteucci M, et al. b-Gammaglutamyltransferase activity in human vulnerable carotid 
plaques. Atherosclerosis 2014;237:307-13.

93. Vasan RS. Biomarkers of cardiovascular disease: molecular basis and practical considerations. Circulation 2006;113:2335-62.

94. Kunutsor SK, Bakker SJ, Kootstra-Ros JE, et al.

Cite this article as: Ndrepepa G, Kastrati A. Gamma-glutamyl transferase and cardiovascular disease. Ann Transl Med 2016;4(24):481. doi: 10.21037/atm.2016.12.27
Circulating gamma glutamyltransferase and prediction of cardiovascular disease. Atherosclerosis 2015;238:356-64.

95. Hill AB. The environment and disease: association or causation? Proc R Soc Med 1965;58:295-300. 